\title{
Circular Patch Antenna Design for Data Centric Cognitive Radio based Networks
}

\author{
Vivekanadam B \\ Professor, \\ Lincoln University, \\ Malaysia. \\ vivekanandam@lincoln.edu.my
}

\begin{abstract}
Inefficient utilization of licensed spectrum bands and overcrowding of unlicensed bands are caused due to the spectrum shortage and growing demand for wireless communication. The wireless spectrum is burdened due to the host centric traditional approaches for data detection and recovery in the IP-based networks that. The service or data is retrieved from the service provider through a new routing path every time the mobile service requester initiates a request. The vacant licensed channels are utilized appropriately enabling opportunistic and efficient band usage of the spectrum using Cognitive Radio (CR) technology. Wireless communication with low cost, compact antenna element, high gain, wideband and low profile can be performed using patch antenna. Patch is a significant aspect of antenna design. The antenna design parameters are understood by varying the patch. A good return loss can be achieved by enhancing the radiation pattern on changing the patch dimensions. High Frequency Structure Simulator (HFSS) is used for simulation and analysis of the circular patch antenna. The return loss, radiation efficiency, Voltage Standing Wave Ratio (VSWR) and radiation pattern of the antenna are analyzed.
\end{abstract}

Keywords: Cognitive radio, circular patch antenna, wireless communication, radiation pattern, High Frequency Structure Simulator;

\section{Introduction}

Fixed policies of spectrum assignments regulates the traditional wireless networks. Only around 15 to $85 \%$ of the assigned spectrum are utilized due to the geographical and temporal variations. Cognitive radio network is an efficient model that enables resourceful utilization and sharing of the spectrum in an productive manner [1]. The cognitive radio network (CRN) enables wireless communication in complex multiuser environment with promising features. CRN aims in facilitation of efficient radio spectrum utilization with high reliability communication for all the network users. Spectrum sensing, management, mobility and sharing are the key functionalities of CRN [2]. At any location and time, the unused spectrum can be sensed by the CR. It can also allocate the user with optimal spectrum band available, based on the spectrum policies and availability. In the presence of a primary user, the CR vacates the spectrum and move to the next optimal spectrum band available. Spectrum sensing can be performed by multi-taper method, cyclo-stationarity method or energy detection method. Artificial intelligence, Machine learning, and wireless network can be integrated in cognitive algorithms.

Figure 1 provides the basic architecture of the cognitive radio network. The routing and MAC protocol components can be adapted dynamically using the cognitive algorithms [3]. Low-cost consumer devices can be used for creating a wide range of waveforms that may be authorized or unauthorized with the extension of the programmability of the radio. Hence network security is a major factor in CR [4]. The network should be able to defend itself from denial of service attacks or intrusion. The data from the network is transmitted or received through the antenna. In large spectrum frequencies, high performance can be maintained using microstrip antennas of low profile, minimal weight and low cost.

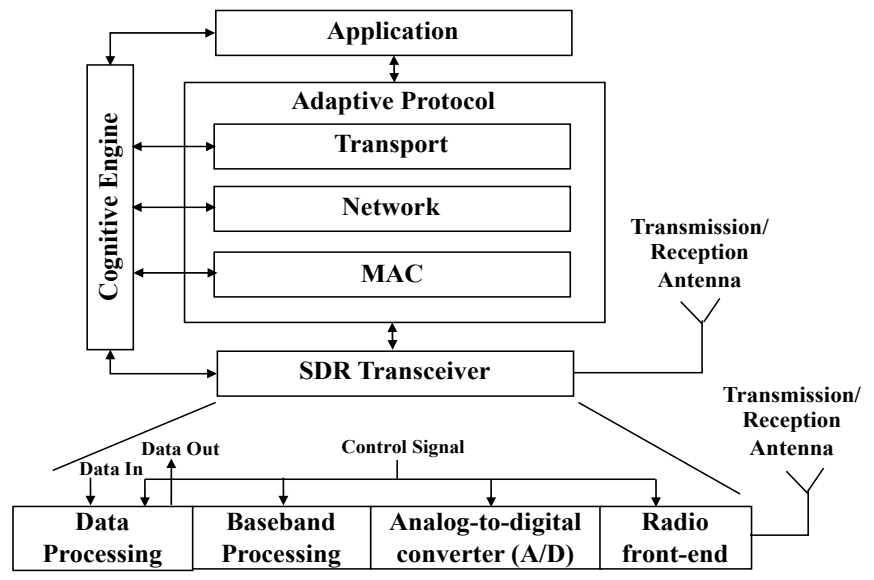

Figure 1. Architecture of Cognitive Radio Network 
Table 1 provides the description of the functions of CRN. Various advantageous features are offered by patch antennas with simple geometric structure. Low fabrication cost, simple, lightweight and extremely low profile antennas can be integrated on printed circuit boards [5]. The impedance, pattern, polarization and operating frequency parameters become versatile on selection of operating mode and shape of the patch [6]. The resonant nature of patch antennas lead to an inherent narrowband functioning which is the major drawback of the patch antennas.

Table 1. Function Table

\begin{tabular}{ll}
\hline \multicolumn{1}{c}{ Function } & \multicolumn{1}{c}{ Action } \\
\hline Packet routing & Packet routing across the network \\
Dynamic spectrum management & Spectrum hole distribution among secondary users is controlled fairly \\
& \\
Transmission power control & User data rate maximization subject to power constraints. \\
Predictive modelling & Spectrum holes availability prediction by employing secondary user \\
Spectrum sensing & Estimation of power contents in the spectrum holes detected. \\
\hline
\end{tabular}

\section{Patch Antenna}

Planar Inverted-F Antenna (PIFA) is a commonly used patch antenna that is in built in cell phones. The specific absorption rate (SAR) of the antenna is good with a quarter-wavelength resonance, thereby decreasing the installation area required on the phone [7]. The omnidirectional pattern and low profile makes this antenna a popular choice. These antennas are derivates of the quarter-wave half-patch antenna. The resonance frequency decreases with the reduction in the length of the half-patch shorting plane [8]. At several cellular bands, the multiple branches of PIFA antennas resonate. The radiation bandwidth characteristics are enhanced using grounded plastic elements in some phones. Better volume reuse characteristics of Folded Inverted Conformal Antenna (FICA) makes it a better choice when compared to PIFA [9].

Different configware code can be loaded into a system enabling its reconfiguration capability. It enables changing the behavior of the system to a certain extent [10]. The runtime and reconfiguration time can be distinguished by static reconfigurability. The dynamic environmental variations may lead to dynamic behavioral changes in the system during runtime. This phenomenon called dynamic reconfigurability. Variation in application services, protocol stacks, radio access technologies and radio spectrum of the wireless equipment and behavioral variations in the wireless networks can be tackled by dynamic reconfigurability in wireless communication [11]. In control engineering, the fault-tolerant control is done using control reconfiguration. Control reconfiguration enables reaching the fault-free system's original control goals on a faulty system. The changing system parameters are matched through variation of far-field pattern, operating frequency and polarization of reconfigurable antenna [12].

Several global research groups like End-to-End Reconfigurability ( $\left.\mathrm{E}^{2} \mathrm{R}\right)$, Wireless Innovation Forum (WINNF), and Wireless World Research Forum (WWRF) have undertaken projects to enhance the wireless communication systems in terms of dynamic reconfigurability. External control can be performed for dynamic variation of the properties of reconfigurable antenna [13]. Signal is received from multiple directions resulting in multiple radiation patterns leading to reconfigurability. In order to design antennas for specific operating frequencies like $2.4 \mathrm{GHz}$ of $\mathrm{WiFi}$ and $3.5 \mathrm{GHz}$ of WiMAX, multiple operating frequencies are required. Limited volume and space are sufficient for reconfigurable antenna. The low profile structure of microstrip patch antennas make them a common target for wireless applications. They are also utilized in satellite communication successfully. Around 6 to $9 \mathrm{dBi}$ maximum directive gain can be provided by a single patch antenna. Lithographic techniques can be used for printing an array of patches over a single substrate [14]. When compared to a single patch, the patch array offers high gain with lesser additional cost.

\section{Proposed Antenna Design}

In this paper, we study the major aspects and features of microstrip patch antennas. Half ground plane is used for calculation of switching between two bands in a rectangular microstrip patch antenna. This enables determining the antenna dimensions for simulation. ANSOFT- High Frequency Structure Simulator (HFSS) is used for simulation study of the microstrip antenna [15]. It is a simulation package for planar antenna design and analysis based on method of movements integrated with full wave electromagnetics. Various parameters like Smith Chart, VSWR, return loss and radiation pattern can be calculated and plotted with this tool. The microstrip antenna (MSA) characteristic parameters can be determined through simulation. Figure 2 provides the antenna design and simulation using HFSS tool. 


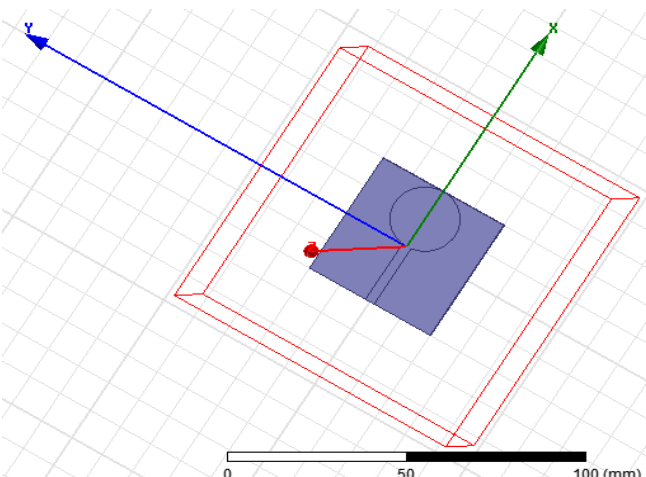

(a)

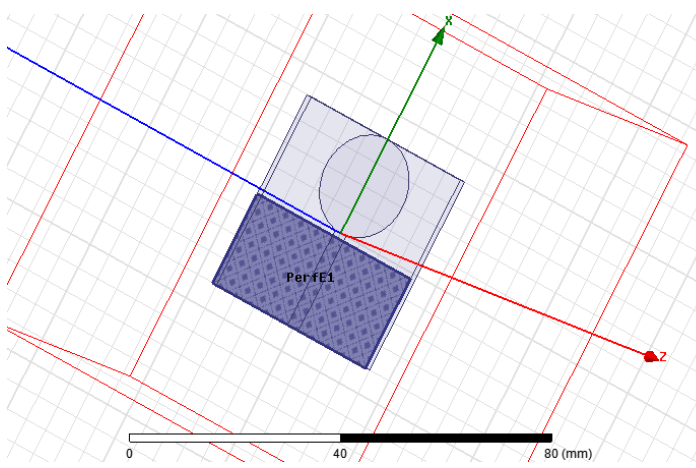

(c)

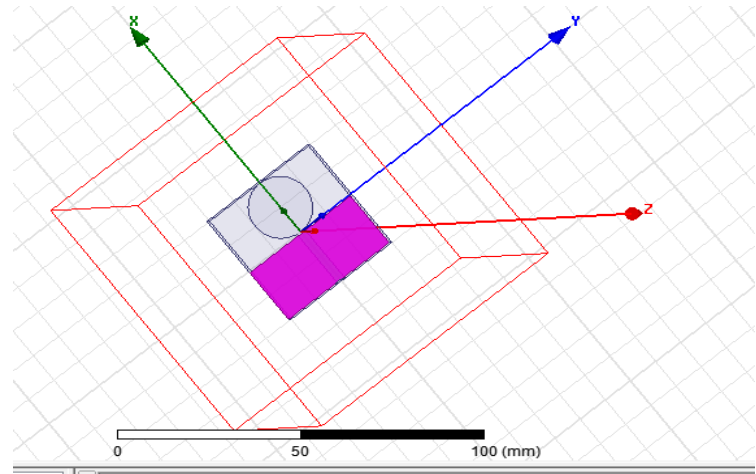

(b)

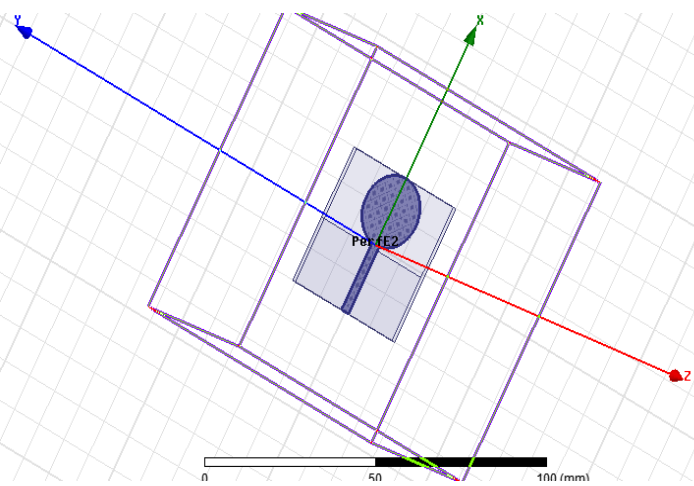

(d)

Figure 2. (a), (b) Antenna with circular patch and feed, (c) Ground plane with excitation, (d) circular patch with excitation

The circular Microstrip Patch Antenna design has three essential parameters namely the operation frequency $\mathrm{f}_{\mathrm{o}}$, substrate dielectric constant $\varepsilon_{\mathrm{s}}$ and dielectric substrate height h. Frequency range between $300 \mathrm{MHz}$ and $24 \mathrm{GHz}$ is used for wireless communication. Operation of the antenna within this frequency range is essential. Hence an appropriate resonant frequency must be chosen for the antenna. This paper uses a resonant frequency of $5.5 \mathrm{GHz}$. FR4 dielectric material with a dielectric constant $\varepsilon_{\mathrm{s}}=4.4$ is selected as the antenna dimension can be reduced using a higher dielectric constant substrate. A height of $1.5 \mathrm{~mm}$ is chosen for the dielectric substrate as the antenna is not supposed to be bulky for use in cellular phones.

\section{Simulation and Results}

The process flow for analysis involves creation of physical environment by defining the options and element types, setting real constants, defining material properties like resistivity, permeability and permittivity and defining a system of units. Further, the geometry is modeled as a solid model and a CAD model and regional attributes are assigned. The model is meshed and boundary conditions are applied. Further, the excitation and load sources are applied in terms of plane wave, magnetic field, current density and port. Finally the model is validated and executed. Figure 3 represents the return loss analysis of the proposed patch antenna.

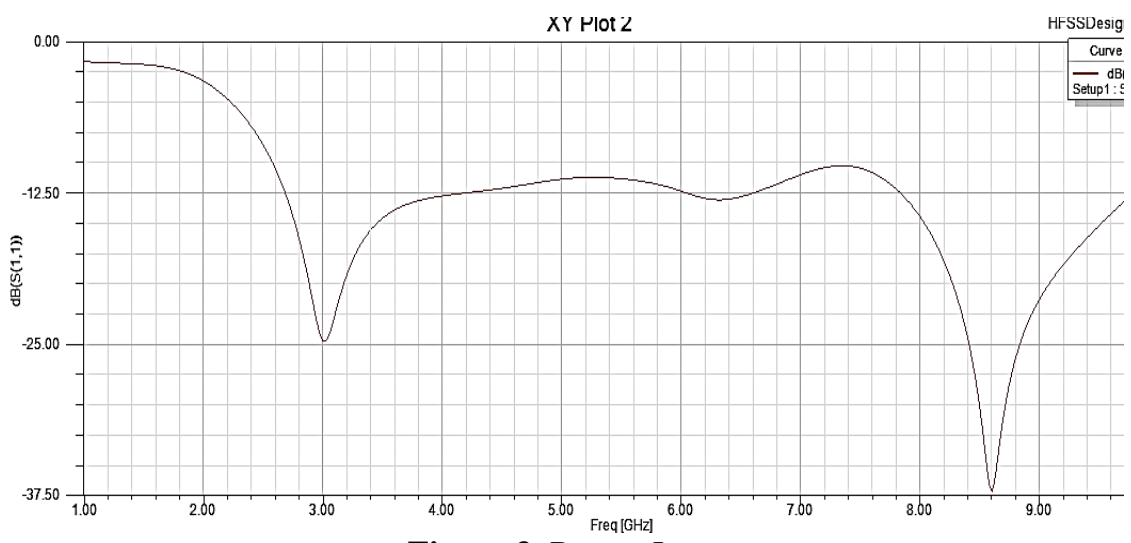

Figure 3. Return Loss 
Post execution, the fields like near field, conducting current, vector and contour are analyzed along with parameters like impedance, current, smith chart, $\mathrm{Z}$ parameter plotting, conversion to $\mathrm{Y}$, and $\mathrm{S}$ parameter. The outer boundary of the circular patch antenna is represented as ABC. The feed dimension along Y axis is $2.86 \mathrm{~mm}$, feed dimension along $\mathrm{X}$ axis is $20 \mathrm{~mm}$, substrate dimension along $\mathrm{X}$ and $\mathrm{Y}$ axis are both $40 \mathrm{~mm}$. The patch radius is $10 \mathrm{~mm}$. Figure 4 provides the VSWR of the proposed antenna. The efficiency of transmission of the radiofrequency power into the load from the power source through the transmission line is represented by the voltage standing wave ratio. Maximum energy transfer represents improved efficiency of the system.

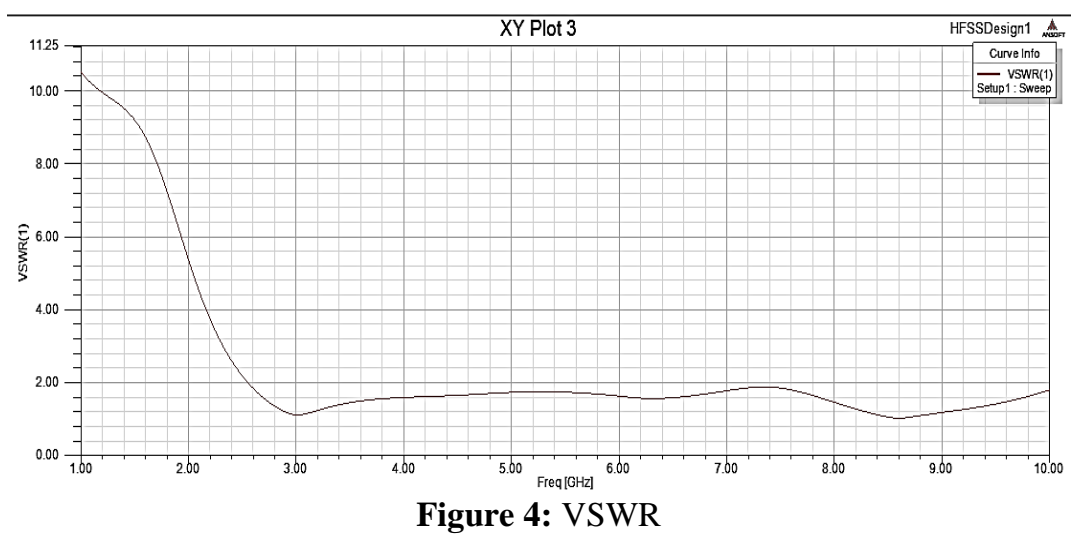

Figure 5 represents the radiation pattern of the antenna. The directivity of the antenna is 6 to $9 \mathrm{dBi}$. When all directivity is transformed to gain, the efficiency of the antenna is $100 \%$. The antenna impedance matching efficiency and radiation efficiency combined together provides the total efficiency. The polarization plane of the antenna refers to the plane where variation of electric field is detected. Similar polarization must be found in both transmitting and receiving antennas for optimal system performance. Circular polarization can be excited in a patch by splitting the signal into equal halves and feeding them to the horizontal and vertical radiator respectively. Further, the phase of one of the signals has to be changed to $90^{\circ}$.

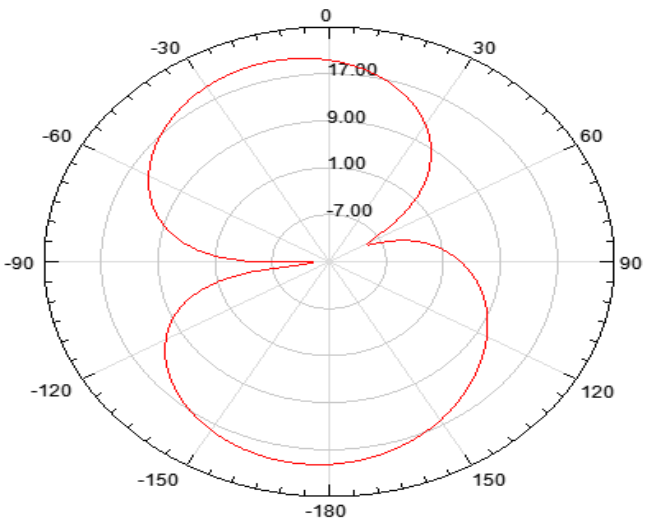

Figure 5: Radiation Pattern

\section{Conclusion}

Cognitive radio multi radio wireless applications can make use of the circular patch antenna. Half ground plane can be used for enabling operation of the designed antenna between two frequencies. The preferred frequency bands can be chosen for operation of the proposed antenna. Design and fabrication of this antenna are simple. For any choice of frequency, satisfactory gain values and good radiation pattern can be obtained. The return loss, radiation efficiency, Voltage Standing Wave Ratio (VSWR) and radiation pattern of the antenna are analyzed. These factors show the efficiency of the proposed patch antenna. Pin diode acts as a switching element for the circular patch antenna. Switching between various states can be achieved using Pin diode though its on-off operation in the ground plane slots.

\section{References}

[1] Alsharif, F., Safi, S., AbouFoul, T., Abu Nasr, M. H., \& Abu-Naser, S. S. (2016). Mechanical Reconfigurable Microstrip Antenna. IAMOT.

[2] Sasipriya, S., \& Vigneshram, R. (2016, December). An overview of cognitive radio in 5G wireless communications. In 2016 IEEE International Conference on Computational Intelligence and Computing Research (ICCIC) (pp. 1-5). IEEE. 
[3] Gupta, M. S., \& Kumar, K. (2019). Progression on spectrum sensing for cognitive radio networks: A survey, classification, challenges and future research issues. Journal of Network and Computer Applications, 143, 47-76.

[4] Hussain, R., \& Sharawi, M. S. (2017). Reconfigurable MIMO antennas for cognitive radios. In Spectrum Access and Management for Cognitive Radio Networks (pp. 233-264). Springer, Singapore.

[5] Valanarasu, M. R., \& Christy, A. (2019). Comprehensive Survey of Wireless Cognitive and 5G Networks. Journal of Ubiquitous Computing and Communication Technologies (UCCT), 23-32.

[6] Sharma, S., Tripathi, C. C., \& Rishi, R. (2017). Impedance matching techniques for microstrip patch antenna. Indian Journal of Science and Technology, 10(28), 1-16.

[7] Karthik, B., Vijayaragavan, S. P., \& Sriram, M. (2018). Microstrip Patch Antenna for Wireless LAN. International Journal of Pure and Applied Mathematics, 118(18), 25-33.

[8] Abirami, M., \& Rajasekar, G. (2016, November). MEMS reconfigurable slotted microstrip patch antenna for cognitive radio application. In 2016 Online International Conference on Green Engineering and Technologies (IC-GET) (pp. 1-4). IEEE.

[9] Singh, T., Ali, K. A., Chaudhary, H., Phalswal, D. R., \& Gahlaut, V. (2018). Design and analysis of reconfigurable microstrip antenna for cognitive radio applications. wireless personal communications, 98(2), 2163-2185.

[10] Rajan, S. P., Paranthaman, M., \& Vivek, C. (2016). Design and Enhancement of Wideband Reconfigurability using Two E-Shaped Patch Antenna. Asian Journal of Research in Social Sciences and Humanities, 6(9), 317-327.

[11] Manjunatha, K. H., \& Mehta, S. (2016, December). Reconfigurable communicating patch antenna for cognitive radio applications. In 2016 International Conference on Control, Instrumentation, Communication and Computational Technologies (ICCICCT) (pp. 461-465). IEEE.

[12] Abirami, M., \& Kashwan, K. R. (2018). Ultra-Wideband Slotted Microstrip Patch Antenna for Cognitive Radio. In International Proceedings on Advances in Soft Computing, Intelligent Systems and Applications (pp. 93-102). Springer, Singapore.

[13] Haoxiang, W. (2019). Multi-Objective Optimization Algorithm for Power Management in Cognitive Radio Networks. Journal of Ubiquitous Computing and Communication Technologies (UCCT), 1(02), 97-109.

[14] Mak, K. M., Lai, H. W., Luk, K. M., \& Ho, K. L. (2016). Polarization reconfigurable circular patch antenna with a C-shaped. IEEE Transactions on Antennas and Propagation, 65(3), 1388-1392.

[15] Mugunthan, S. R. (2020). Novel Cluster Rotating and Routing Strategy for software defined Wireless Sensor Networks. Journal of ISMAC, 2(02), 140-146. 